\title{
An Appraisal of Diverse Image Augmentation Methodologies
}

\author{
M. S. Sonawane, C. A. Dhawale
}

\begin{abstract}
Image augmentation is very significant, challenging methods in image exploration. The intention of augmentation of the image is to expand the graphical image form, or to give a superior renovated illustration for imminent programmed image treating. Various images such as aerial images, images of satellite, medical images, etc., undergo for noise and meager contrast. Therefore, it is obligatory to eliminate the noise and to augment the contrast in order to upsurge image superiority. Among imperative phases in medical images exposure and investigation is an image development practice that develops the worth or lucidity of images for hominoid seeing, eliminating noise and blurring, rise contrast, and enlightening minutiae are cases of enrichment maneuvers. The augmentation practice varies from one arena to another rendering to its purpose. The present methods of image augmentation could be divided into 2 groupings: frequency, spatial domain enrichment. This work offers a brief summary of image augmentation methods in spatial territory. More precisely, paper classifies processing methods centered representative techniques of Image augmentation. Hence an impact of this paper is to categorize and analyze image augmentation methods, effort for evaluation of inadequacies and common desires in an arena of dynamic investigation and at last paper focuses on auspicious information on exploration for image augmentation for imminent study.
\end{abstract}

Keywords: Image augmentation, spatial, frequency domain, histogram equalization.

\section{INTRODUCTION}

Image augmentation could be expressed by means of assumed an input poor class image, the production great superiority image for accurate uses. The ambition is towards increase the pictorial form of given image, to offer a healthier renovate depiction for forthcoming computerized image handling, like analysis, segmentation, feature extraction, classification, recognition, etc. Likewise, it supports to scrutinize the background data, which is crucial to know object actions short of demanding costly humanoid optical examination. Doing image improvement considerate under poor superiority image is a stimulating question since these causes. Because of low contrast, substances cannot obviously mine from the dusky contextual. Maximum color centered approaches can miscarry on such difficulty whenever the color of the substances, background are analogous. The review of existing procedures is founded on the present systems of image augmentation that could be ordered into 2 comprehensive sorts: spatial domain, frequency province image augmentation.

Revised Manuscript Received on April 21, 2020.

* Correspondence Author

Manojkumar Sahebrao Sonawane*, Ph.D., computer science in SGBAU, Maharashtra

Dr. Chitra A. Dhawale, Ph.D., computer science in SGBAU, Maharashtra

(C) The Authors. Published by Blue Eyes Intelligence Engineering and Sciences Publication (BEIESP). This is an open access article under the CC BY-NC-ND license (http://creativecommons.org/licenses/by-nc-nd/4.0/)
The spatial territory image improvement works straightly on the pixels.The chief benefit of the spatial territory system is, they abstractly artless to know and the complication of such methods are stumpy that favors factual time executions. However, these procedures are usually inefficient to provide satisfactory healthiness and noiselessness necessities. Frequency territory image improvement is a terminology employed to designate the study of signals or mathematical utilities related to frequency, function right on image transform constants, like Fourier Transform (FT), Discrete Cosine Transform (DCT), Discrete Wavelet Transform (DWT), Discrete Fourier Transform (DFT), etc. The pluses of frequency image enrichment comprise little complication of calculations, effortlessness of seeing, handling the frequency structure of the image, relaxed use of distinct transmuted area belongings. The elementary restrictions, comprising are it can't concurrently improve whole fragments of the image too fine; it's moreover hard to mechanize the image augmentation method. In this work, conferring to if boosted image implant great eminence background data; the present methods of image improvement such as spatial field approach could yet once more be categorized into 2 wide types: spatial filter, point processing operation. Customary approaches of an image augmentation are; to advance lower superiority image themselves. This not embeds great superiority background data. The cause is in the dusky image, certain regions are too dusky that whole data is at present vanished in such areas. No trouble exactly how considerable brightness enrichment applied, it couldn't be competent to get back missing data. Frequency territory systems could yet once more be ordered into 3 groups: Image sharpening, Image smoothing, periodic noise lessening through frequency area filtering. Work focuses on improvement of image seeing parts of spatial domain enhancement methods. The remaining work is ordered as below. Section 2 offers a short review of correlated literature, in Section 3 analysis of spatial domain techniques is deliberate; Section 4 is about some frequency domain methods. Usefulness and annotations are written in section 5, 6 respectively. The result, conclusion is mentioned in Section 7 and 8 correspondingly.

\section{LITERATURE REVIEW}

Image augmentation course comprises of an assortment of practices that pursue to advance the visual look of a given image, to renovate given image in a format well suitable for examination by a humanoid, appliance. The basic purpose of image enrichment is to adapt an image individuality to mark this well right for an assumed job, precise spectator. In the course of this practice, one or other feature of given image are amended.

Published By:

Blue Eyes Intelligence Engineering DOI: 10.35940/ijeat.D7955.049420

Journal Website: www.ijeat.org

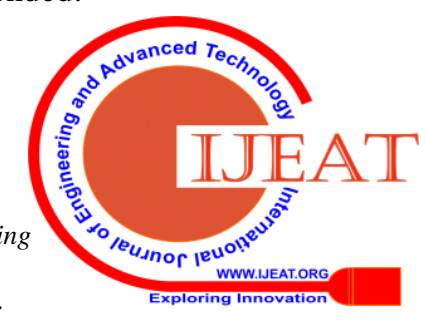


Digital image development methods offer a mass of varieties for enlightening the pictorial excellence of the images. Applicable selection of these methods is significantly inclined by the imaging modality, assignment present, observing circumstances. It's imperative to retain in notice that augmentation is an exact particular expanse of image handling. Expansion in the superiority of corrupted images could be attained by exhausting augmentation methods request. The efforts made through several investigators on behalf of image improvement are deliberated in his work, Madhu[1] recommended adaptive histogram equalization formed a grander consequence, nevertheless the image is yet not unrestricted from splashing out form. The perspicacity is pitiable, the background data, plane has been yet fuddled and miserable in disparity. Alpha rooting condensed whole image into dusky tone. Although clouds skeleton that was observable in occasion of the histogram equalization is vanished. Agaian [2] advised, the shared no transform based improvement system is total histogram equalization that tries in the direction of modify the spatial histogram of image to diligently tie a constant spreading. Histogram equalization undergoes from the difficulty of being weakly appropriate for holding confined aspect because of its inclusive handling of the image. Tang [3] advocated overall equalization of histogram that standardizes the intensity histogram towards estimated constant circulation. In reality, the global histogram amendment delights all sections of the image similarly and, therefore, habitually produces meager local presentation in the form of detail safeguarding. Hence, numerous native image enrichment procedures are presented to expand development.

\section{SPATIAL DOMAIN METHODS}

These methods unswervingly compact on the image pixels. Spatial territory ways, such as histogram equalization, power law transforms, logarithmic transforms, etc., are centered on the straight influence on the image pixels. These procedures are predominantly convenient for openly modifying discrete pixels gray level prices, therefore whole disparity of the complete image. Conversely, these methods, improve the entire image in a constant way that in several circumstances yields objectionable consequences. This is difficult to selectively mend edges, former essential information successfully. Approaches such as equalization of histogram are operational at numerous images. Methodologies could be categorized in 2 groupings: spatial filter, point processing operation. Summary of few of the renowned approaches is conversed here. Point processing maneuvers are the artless spatial area maneuver since actions are executed only on a solitary pixel. Output image pixel values rest on input image pixel values. The Point handling tactics could be categorized in 4 types for example image negatives where gray level values of image pixel are reversed with aim to acquire a corresponding negative image. Assume eight bit image with dimension $\mathrm{M} \times \mathrm{N}$, at point every pixel value in the imaginative image is subtracted from 255. Such images are convenient for increasing gray, white factor, rooted in dusky areas of an image. The alternative method is an image thresholding transformation where suppose $r$ is threshold cost in input image. Image thresholding methods are predominantly convenient in dissection of image to separate interested image apart from the background. Log transformation, which draws constricted variety of little gray levels in a broader assortment of gray levels means magnify perky pixel values, wrapping dusky pixel values. The inverse logarithmic revolutions draw a widespread series of gray level beliefs in a contracted variety of beliefs of gray level, that means magnify dusky pixel values and wrapping perky pixel values. These processes are predominantly utilized as soon as image gray level values have tremendously outsized variety and lesser series correspondingly. Logarithmic transformations could be employed to improve the image intensities such as gamma revolution, where the value of gamma less than one. Generally, this is employed to upsurge the disparity of poorer intensity values or detail. These methods are exclusively advantageous for getting fact in the Fourier transforms. There exist 3 kinds of piecewise linear transformations: bit plane slicing, contrast stretching and intensity level slicing. Contrast broadening is image improvement practices, which comprise treating an input image to create it superior to humanoid spectators. This technique is typically utilized for post handling by altering dynamic range or contrast or equally in image. This course increases the disparity by widening the gray level value series to span an anticipated gray level value series. Such revolution is similarly termed as normalization or image intensity renovation. Present methods for disparity improvement could be yet once more time separated into 2 clusters: direct, indirect approaches. Direct approaches express a contrast extent, attempt to develop it. Indirect approaches develop the contrast by using the underutilized areas, the dynamic variety lacking outlining particular contrast tenure. In work, disparity expansion ways could be roughly divided in two collections: Histogram Equalization abbreviated as HE, Tone Mapping. HE is frequently utilized approach for contrast improvement. The key purpose of such technique is to attain a constant scattered histogram. Rewards of this method comprise this one undergoes from the difficulty of being weakly appropriate for holding native element because of its universal action of image minor facts Drawback is it's unsuitable in certain uses like user electronic yields, where clarity conservation is essential towards escape maddening objects. The equalization consequence is typically an inappropriate damage of pictorial information of superiority, of the intensity scale. The histogram specification method is an alternative tactic for improvement of contrast. In such technique, the histogram outline is indicated by hand afterwards a transformation job is built. Table 1 displays short study of histogram augmentation systems. The tone mapping is an alternative tactic for disparity improvement practices. At such technique if one desires to output great dynamic variety image on display or paper, one should somehow translate the comprehensive intensity assortment in the image to the poorer series sustained by the demonstration. This method is carried into logarithmic scale, in luminance station. This method is exploited to alter floating point radiance map in eight bit demonstration of rendering solicitations. The 2 key purposes of this methodology are image details conservation and offering sufficient entire luster data in short dynamic assortment image. 
Gray, intensity level carving is one more practice of the piecewise linear revolution. At this technique, intensity level carving climaxes definite variety of gray levels in the input image. Such revolutions allow dissection of particular gray level areas of the remaining image. The bit plane carving is one more type of the piecewise revolution that climaxes an impact prepared for entire image presence by certain bits consumed for the pixel gray levels. The spatial filter tasks are executed onto the pixel along his direct neighbors. These procedures are similarly known as neighborhood maneuvers. Depending on the kind of actions made onto pixels, these strainers are categorized in 2 classes: linear spatial filters and nonlinear spatial filters. First practice encompasses convolve a cover by image that is fleeting weighted façade above complete image. Such cover is likewise termed for example kernel or a window or a template. On the other hand, nonlinear spatial filters are the filters where improved image isn't linearly associated with the pixels in input image neighborhood.

\section{FREQUENCY DOMAIN METHODS}

These methods are appropriate for treating an image conferring towards frequency information. Standard with these methods comprises of calculating a 2D discrete unitary renovate of an image for illustration 2D DFT, operating transform constants through an operator, execution contrary renovate. An orthogonal renovation of an image consumes 2 modules; namely magnitude and another is phase. The former entails information of image frequency. Second is utilized toward reestablish an image again to spatial territory. Common orthogonal renovates are DCT, DFT, Hartley Transform, etc. The transmute field allows action on image frequency data, hence great frequency data like edges, further delicate material could be effortlessly boosted [4]. The filtering idea is informal towards imagine in frequency sphere. So, augmentation of the input image could be prepared in frequency territory established on the DFT. It's predominantly suitable in the convolution when spatial range of point spread structure is bigger than convolution concept.

\section{USEFULNESS}

Image augmentation is employed for increasing image superiority. The uses of image enrichment are in satellite, medical imaging, remote sensing, aerial imaging, etc. Such methods are utilized in several regions like astrophotography, forensics, and fingerprint matching and so on. These methods when functional to pictures, videos assist the visually weakened in reading small print, using television and computers, and face recognition. Brightening, sharpening, contrast enhancements are few methods utilized to create the images rich. In the e-learning area, image enrichment is employed to spell out the substances of chalkboard as watched in spilled video. The medical imaging habits it on behalf of falling noise, refining particulars to mend the pictorial illustration of the image. This marks image enhancement an obligatory sustaining tool for reviewing x-rays, ultrasound, etc. In case of forensics, image enrichment is utilized for credentials, proof assembly, investigation, etc. Images attained from criminality scene investigations, security video analysis, fingerprint recognition, etc., are improved to assist in the protection of sufferers, identification of wrongdoers, etc.

Table- I: Short Study of Histogram Enhancement Methods

\begin{tabular}{|c|c|c|c|}
\hline Writer & Model & Treating Methods & Uses \\
\hline Agaian SS [5] & $\begin{array}{l}\text { Histogram Equalization centered } \\
\text { Logarithmic renovate }\end{array}$ & $\begin{array}{l}\text { The log lessening zonal } \\
\text { degree method; Logarithmic } \\
\text { renovate histogram shifting }\end{array}$ & $\begin{array}{l}\text { Safety Investigation, } \\
\text { Movement checking }\end{array}$ \\
\hline Наo Hu [6] & $\begin{array}{l}\text { Information adaptive video handling } \\
\text { standard }\end{array}$ & $\begin{array}{l}\text { Taxonomy of content, } \\
\text { adaptive treating }\end{array}$ & Machine Learning \\
\hline Tarik A. [7] & $\begin{array}{lll}\begin{array}{l}\text { Histogram } \\
\text { adaptation }\end{array} & \text { Equalization } & \text { centered } \\
\end{array}$ & $\begin{array}{l}\text { Histogram amendment } \\
\text { structure, content adaptive } \\
\text { procedure }\end{array}$ & $\begin{array}{l}\text { LCD display device; } \\
\text { Low quality video }\end{array}$ \\
\hline Sangkeun Lee [8] & Dynamic series density & Retinex theory, DCT & $\begin{array}{l}\text { Image/video } \\
\text { compressing }\end{array}$ \\
\hline Viet Anhnguyen [9] & $\begin{array}{lll}\text { Cauchy distribution } & \text { model; } & \text { AC } \\
\text { transform coefficient }\end{array}$ & $\begin{array}{l}\text { Video restored from several } \\
\text { compressed copies of video } \\
\text { content }\end{array}$ & Video Compression \\
\hline R.C. Gonzalez [10] & Histogram Equalization & $\begin{array}{l}\text { Comprehensive Histogram } \\
\text { Equalization }\end{array}$ & $\begin{array}{l}\text { Video, Image Safety } \\
\text { Investigation }\end{array}$ \\
\hline Xuan Dong [11] & Image Reversing Model & $\begin{array}{l}\text { Dehaze algorithm, } \\
\text { Reversing the input stumpy } \\
\text { illumination video }\end{array}$ & $\begin{array}{l}\text { Medical imaging, } \\
\text { Movement checking }\end{array}$ \\
\hline Shan Du [12] & $\begin{array}{l}\text { Adaptive Region } \\
\text { Equalization model }\end{array}$ & $\begin{array}{l}\text { Adaptive Region centered } \\
\text { Technique }\end{array}$ & $\begin{array}{l}\text { Acknowledgment } \\
\text { Face }\end{array}$ \\
\hline A.A Wadud M [13] & Dynamic HE (DHE) & DHE method & $\begin{array}{l}\text { Poor eminence video, } \\
\text { Medical Image }\end{array}$ \\
\hline Boudraa A.O [14] & 2DTKEO model & 2DTKEO & Satellite image etc. \\
\hline David M. [15] & Multi histogram equalization model & MHE approaches & Image processing \\
\hline
\end{tabular}




\begin{tabular}{|l|l|l|l|}
\hline Sara Hashemi [16] & Improved Histogram Equalization & Genomic procedures & $\begin{array}{l}\text { Calculate high dynamic } \\
\text { series image processing }\end{array}$ \\
\hline George D [17] & $\begin{array}{l}\text { Improved Histogram Equalization and } \\
\text { Histogram Specification }\end{array}$ & $\begin{array}{l}\text { Histogram centered image } \\
\text { improvement }\end{array}$ & Image processing \\
\hline
\end{tabular}

\section{ANNOTATIONS}

The point processing approaches are very basic; however necessary image handling ways are utilized predominantly aimed at disparity improvement. A negative of image remains appropriate aimed at increasing white factor, rooted into dusky constituencies besides used into the medical imaging. For broad resolution, contrast operation power law transformations are convenient. In case of dark image, spreading out of gray levels is proficient by employing a power law revolution by means of minuscule proponent. The log revolution remains advantageous aimed at improving detail into duskier image areas by overhead of factor into bright areas upper level beliefs. Aimed at image consuming a washedout form, gray level density remains achieved by means of the power law revolution through value of $\gamma>1$. Image histogram affords significant information about image contrast. Histogram equalization is a revolution, which expanses the disparity by reorganizing values of gray level regularly.

\section{RESULTS}

In this work, different approaches of image enhancement are considered; the summarization of different enhancement methods functional on digital image processing is discussed briefly. Procedures of image enhancement specified in this work are exploited in various recent machines for identification of image, face, recognition of pattern, etc. Image enhancement has an auspicious and challenging opening as the general enhancement process and is center of kindness of present investigation. Here is no single system which could be assumed excellent for all image types. Every system has its advantages, disadvantages, uses. Not each system evenly acceptable for all image type. Because of such concerns, image enhancement remains a challenging thing in image processing and is quiet expecting problem.

\section{CONCLUSION}

Image augmentation methods offer a widespread tactic for amending images to accomplish pictorial tolerable images. The selection of these methods is a role of the particular assignment, image data, spectator features, and seeing environments, etc. The analysis of image augmentation methods, particularly in a spatial territory is done and is very challenging, significant elements of digital image handling. The fallouts for every technique are likewise conferred in this paper. Depending on the image type, noise type with which it's despoiled, a minor amendment in discrete technique or amalgamation of some approaches supplementary mends pictorial excellence. This work focuses on study the present methods of image augmentation that could be divided into 2 wide groups as frequency, spatial territory augmentation. The paper revealed existing methods of image augmentation and chat the benefits and drawbacks of those procedures. Although the paper did not confer reckoning charge of augmentation processes it may show the vital share while electing the process aimed at actual uses. The paper likewise has explained modern approaches of image augmentation and pointed auspicious instructions on exploration for image augmentation in spatial territory for imminent study. The impending opportunity could be the growth of adaptive procedures for operative image improvement by means of diverse approaches.

\section{REFERENCES}

1. Arun R, Madhu S. Nair, R. Vrinthavani and Rao Tatavarti. “An Alpha Rooting Based Hybrid Technique for Image Enhancement”, online publication in IAENG, 24th August 2011.

2. Agaian, SOS S., Blair Silver, Karen A. Panetta, "Transform Coefficient Histogram-Based Image Enhancement Algorithms Using Contrast Entropy", IEEE Transaction on Image Processing, Vol. 16, No. 3, March 2007.

3. Jinshan Tang Eli Peli, and Scott Acton, "Image Enhancement Using a Contrast Measure in the Compressed Domain", IEEE Signal processing Letters, Vol. 10, NO. 10, October 2003.

4. S.S. Bedi and Rati Khandelwal, "Various Image Enhancement Techniques- A Critical Review", International Journal of Advanced Research in Computer and Communication Engineering Vol. 2, Issue 3, 2013.

5. S. S. Agaian, S. Blair and K. A. Panetta, "Transform coefficient histogram-based image enhancement algorithms using contrast entropy", IEEE Trans. Image Processing, vol. 16, no. 3, pp. 741-758, 2007.

6. H. Hu, "Video enhancement: content classification and model selection", Ph. D. Thesis, Technique Universities Eindhoven, Eindhoven, Netherlands, 2010.

7. Tarik Arici, Salih Dikbas, "A Histogram Modification Framework and Its Application for Image Contrast Enhancement", IEEE Transactions On Image Processing, Vol. 18, NO. 9, 2009.

8. S. Lee, "An efficient content-based image enhancement in the compressed domain using Retinex theory", IEEE Trans. Circuits and Systems for Video Technology, vol. 17, no. 2, pp. 199-213, 2007.

9. V. A. Nguyen, Y. P. Tan and Z. H. Chen, "On the method of multicopy video enhancement in transform domain", Proc. of the 16th IEEE International Conference on Image Processing, pp. 2777- 2780, 2009.

10. R. C. Gonzalez and R. E. Woods, Digital Image Processing, Prentice Hall, New Jersey, 2008.

11. X. Dong, Y. Pang and J. Wen, "Fast efficient algorithm for enhancement of low lighting video", Proc. Of the $37^{\text {th }}$ International Conference and Exhibition on Computer Graphics and Interactive Techniques, 2010.

12. Shan Du, Ward R.K., "Adaptive Region-Based Image Enhancement Method for Robust Face Recognition under Variable Illumination Conditions", IEEE Transactions on Circuits and Systems for Video Technology, Volume: 20, Issue: 9, Page(s): 1165 - 1175, IEEE, 2010.

13. A. A. Wadud, M. Kabir, M. H. Dewan and M. C. Oksam, "A dynamic histogram equalization for image contrast enhancement”, IEEE Trans. Consumer Electronic, vol. 53, no. 2, pp. 593-600, 2007.

14. A. O. Boudraa and E. H. S. Diop, "Image contrast enhancement based on 2D teager-kaiser operator", Proc. of the IEEE International Conference on Image Processing, pp. 3180-3183, 2008.

15. David Menotti, Laurent Najman, Jacques Facon, and Arnaldo de A Araújo, "Multi-Histogram Equalization Methods for Contrast Enhancement and Brightness Preserving", IEEE Transactions on Consumer Electronics, Vol. 53, No. 3, 2007.

16. S. Hashemi, S. Kiani, N. Noroozi and M. E. Moghaddam, "An image contrast enhancement method based on genetic algorithm", Pattern Recognition Letters, vol. 31, no. 13, pp. 1816-1824, 2010. 
17. George D and J. Yagnik, "Automatic, efficient, temporally-coherent video enhancement for large scale applications", Proc. of the 17th ACM International Conference on Multimedia, pp. 609-612, 2009.

\section{AUTHORS PROFILE}

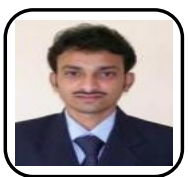

Manojkumar Sahebrao Sonawane, have completed M.Sc.(Computer Science) in 2005, MCA in 2012. Qualified SET in year 2015, NET in 2019. Currently pursuing Ph.D. in computer science in SGBAU, Maharashtra under supervision of Dr.C.A.Dhawale. Have publications in IEEE, Springer, IJITEE, IJPT, IJCA, IJSR etc., conference, journal, and proceedings. Area of interest is image processing. Research work is on optical character recognition. Have 13 years of teaching experience in the computer field. Participated in several conferences, workshops, seminars, faculty development programs, poster presentation competitions etc. Take part in syllabus framing workshops. Organized various workshops, seminars, and guest lectures. Worked as coordinator of the CSI student branch. Awarded with second price for university and state level research activity Avishkar-2009.

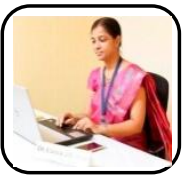

Dr. Chitra A. Dhawale. Awarded Ph.D (Computer Science) in 2009. Subject specialization is image processing. Total, 21 years teaching experience. Papers published in national journals-02, international journals19. Papers presented in National conferences-19, International conferences-26. Ph.D. guide of computer science in SGBAU, Maharashtra. 3 book chapters are in IGI Global. Filed patent. Professional memberships are life member ISTE, New Delhi, senior member of the IACSIT, Member of International Association of Engineers (IAENG), Hong Kong. 1 proposal submitted to DST. Interaction with professional institutions like International Journal of Computer Application, International Journal of Electronics and Electrical Engineers, International Journal of Innovative Research in Information Security, International Journal of Advancements in Computing Technology, International Journal on Advances in Information Sciences and Service Sciences, International Journal of Computer Science \& Systems, International Journal of Engineering and Advanced Technology, Covenant Journal of Computer Science, Vietnam, Bio-Info Publications etc.

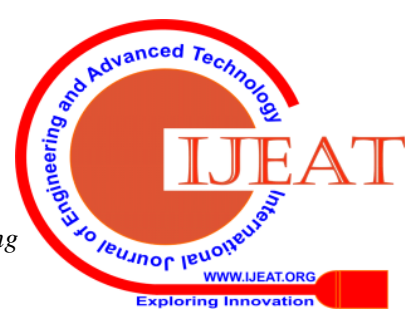

